\title{
Conceptualization and Rehabilitation of Executive Functions
}

\section{A Review of the Literature}

\author{
Artemisa Rocha Dores, ${ }^{1,2}$ Irene Palmares Carvalho, ${ }^{3}$ Fernando Barbosa, ${ }^{4}$ \\ Claúdia Martins, ${ }^{5}$ Liliana de Sousa, ${ }^{1}$ and Alexandre Castro-Caldas ${ }^{6}$ \\ ${ }^{1}$ Institute of Biomedical Sciences Abel Salazar, University of Porto (ICBAS-UP), Portugal, \\ ${ }^{2}$ School of Allied Health Sciences, Polytechnic Institute of Porto (ESTSP-IPP), Portugal, \\ ${ }^{3}$ School of Medicine, University of Porto (FMUP), Portugal, ${ }^{4}$ Faculty of Psychology and \\ Educational Sciences, University of Porto (FPCEUP), Portugal, ${ }^{5}$ Learning Disability \\ Directorate, Abertawe Bro Morgannwg University Health Board, Port Talbot, UK, \\ ${ }^{6}$ Institute of Health Sciences, Portuguese Catholic University (ICS-UCP), Porto, Portugal
}

\begin{abstract}
Publications on executive functions have increased in the last few years, reflecting the importance of this area of study. The scientific output on executive functions is now extremely diverse, comprising variations around terminology, assessment, and rehabilitation practices, and ranging in topics from neuroanatomical correlates of executive functions to effects of executive dysfunction. This review seeks to explore this diversity around executive functions in order to provide an integrative overview of the topic that systematizes the current knowledge in this area, and to point to trends and future directions for research and practice. The literature review was conducted in the ISI Web of Knowledge databases. The analysis was conducted in NVivo9. Two independent coders applied an inductive analysis to all relevant papers, building a hierarchical model with categories and subcategories of themes emerging from the literature. A confirmatory analysis followed, with the same independent coders applying the model to the papers. The process was validated by a third expert researcher. Out of 187 titles and abstracts, 91 were analyzed. The outcomes were structured in six main categories: central nervous system, diagnosis, population, assessment, intervention, and theoretical models. Key findings included promising trends in executive function assessment and rehabilitation as well as potential implications for current health approaches and future research.
\end{abstract}

Keywords: executive function, brain injuries, neuropsychological patients, neuropsychological recovery, review

The number of publications on executive functions (EF) has increased in the last few years, which reflects the importance and complexity of this area of study. The scientific output on EF is now extremely diverse, comprising many variations around terminology, assessment, and rehabilitation practices, and ranging in topics from neuroanatomical correlates of EF to effects of executive dysfunction.

Lezak coined the term "executive function," presenting it as involving skills to formulate goals, to plan strategies to achieve those goals, and to self-evaluate one's performance during these activities (Lezak, 1982, 1987). However, the construct and its definition are still complex and unclear, which is reflected, for example, in the diversity of terms that can be found in the literature to designate executive functioning (Fuster, 1997; Pennington, 1997; Roberts \& Pennington, 1996; Stuss \& Benson, 1984, 1986).

Despite the wide range of views about this functioning, there is increasing consensus about the fact that EF are 
not a unitary construct (Robbins, 1996). This assumption gathers support from clinical, pathophysiological, and functional neuroimage evidence (Masterman \& Cummings, 1997), though a central executive, that could be the executive attention system or working memory, has recently been suggested to provide some unity to EF (McCabe, Roediger, McDaniel, Balota, \& Hambrick, 2010).

Considering the nature and diversity of EF subprocesses, there are various difficulties associated with their assessment and rehabilitation. One such difficulty has to do with the ecologic validity of the instruments used to measure EF - traditionally, neuropsychological tests. The instruments to be used and the proposal of alternative methods of assessment are frequently addressed in the literature.

EF rehabilitation is another major concern. When EF are damaged, other mental functions tend to become affected, producing general cognitive and behavioral disorganization. Executive dysfunction, or dysexecutive syndrome, can be quite pervasive and disabling to patients and their families (Baddeley, 1986; Baddeley \& Wilson, 1988). In these cases, neuropsychological rehabilitation aims at "shifting the individual from a more dependent, externally supported state to a more independent and selfregulated state" (Mateer, 1999, p. 50). This implies not only the transfer, but also the generalization of the acquired abilities to real life.

The role and functioning of the prefrontal cortex have been central to the understanding of EF (Bechara, Damasio, \& Damasio, 2000; Luria, 1973, 1980). The prefrontal cortex is regarded as a heteromodal association area, connected with cortical and subcortical regions (TirapuUstárroz, García-Molina, Luna-Lario, Roig-Rovira, \& Pelegrín-Valero, 2008a), in which specific contributions are attributed to different functional circuits. A variety of theories or models have offered different conceptualizations of the role of prefrontal regions in EF, provided functional explanations for the processes underlying these functions and/or facilitated the study of the complex relationship between $\mathrm{EF}$ and behavior. In two recent works, the authors presented a review of the main existing theoretical models (e.g., hierarchical model, working memory and executive functions model, hypervigilant attentional system, somatic marker model, integrative model, dynamic filter theory, theory of cognitive complexity and control, model of differential axis in the executive control, hypothesis of the frontal lobes' hierarchical representation, supervisor attentional system, model of attentional control, rostrolateral prefrontal cortex model, and Christoff and Burgess's models) (TirapuUstárroz et al., 2008a, 2008b). This review demonstrates the complexity of EF and the efforts to understand their nature.

This review seeks to explore the diversity around EF in scientific literature and to provide an integrative overview of this topic that systematizes the current knowledge in terms of the themes that emerge from the literature. This systematization will allow quick access to the vast field of EF. Additionally, its goal is to point to trends and directions for research and practice in future works.

\section{Material and Method}

General stages and protocols of this study's design follow suggestions by Creswell (2003). A systematic literature review of scientific papers indexed in the ISI Web of Knowledge databases until April, 2011, was conducted. Keywords and search string were: (executive dysfunction* OR dysexecutive function* OR dysexecutive syndrome OR executive function*) AND ("traumatic brain injury" OR TBI OR "acquired brain injury" OR ABI OR "neurologic* diseases" OR "neurologic* disorders") AND (model* OR review).

A total of 187 abstracts were found between 1994 and 2011. Exclusion criteria were: (1) topics outside of the scope of this study's goal, such as immunology; (2) languages other than English; and (3) documents from meetings. After the application of the exclusion criteria, 91 records were retained for analysis.

These articles were exported to an Endnote library and then exported to NVivo 9 (2011, QSR, Victoria). The analysis was conducted on the 91 papers' titles and abstracts. This procedure was meant to ensure the emergence of the broadest possible scope of relevant subthemes in this study. Once we establish the state of the art in the field of EF, relevant questions may be formulated to be pursued in a deeper and more focused way in future studies. First an inductive analysis was conducted to identify key concepts. Two reviewers built a hierarchical tree of their respective codes. Similar codes of the two reviewers were merged, and a preliminary model was built describing the nature and quality of the information. Then, findings were obtained comparing the analysis of the two reviewers and reaching consensus, with a final validation by the third, independent expert researcher. The final model was then constructed.

In order to validate the original coding, the two reviewers used the developed framework and applied it to re-code all the material. The re-coding was conducted independently. The goal was to test the robustness and external validity of the previously developed framework. It also allowed to check the internal validity and to reach an agreement over both construct and concept validity of the hierarchical tree.

Inter-rater level of agreement was 0.86 for Cohen's kappa coefficient. The agreement between the two reviewers (mean of agreement in all categories) was of 96.71 $(S D=10.35)$. Final data were analyzed through descriptive statistics.

\section{Results}

\section{Scientific Output}

Figure 1 shows that the number of publications on EF has been increasing over the past years which indicates the current interest in the topic. 


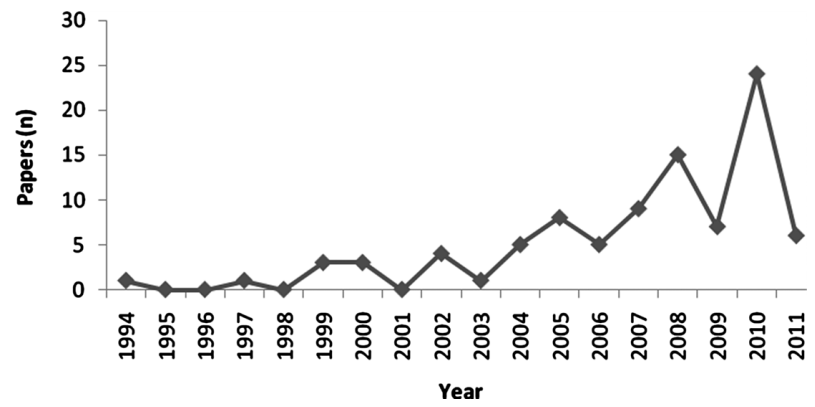

Figure 1. Number of EF papers by year.

\section{Main Themes (NVivo Model)}

Papers varied in terms of article types and scientific domain (see Table 1). The main themes emerging from the literature were, in descending order: central nervous system ( $n=86$ records; $95 \%$ of all), diagnosis ( $n=82$ records; $90 \%$ of all), population ( $n=72$ records; $79 \%$ of all), assessment $(n=61$ records; $67 \%$ of all), intervention $(n=54$ records; $59 \%$ of all) and theoretical models ( $n=41$ records; $45 \%$ of all) (see Tables 2 and 3 ).

\section{Article Types}

51 records are literature review articles $(56 \%)$ and 40 are empirical articles (44\%).

\section{Scientific Domain}

82 articles fall within the area of neuropsychology (90\%), 25 within neurosciences (27\%), 9 within social sciences (10\%), 2 within neuropsychiatry (2\%), 1 within psychology $(1 \%)$, and one refers to other areas $(1 \%)$.

Table 1. Paper variation in terms of article types and scientific domain

\begin{tabular}{lccc}
\hline & $\begin{array}{c}\text { No. of } \\
\text { records }\end{array}$ & $\begin{array}{c}\text { \% in the } \\
\text { (sub)category }\end{array}$ & $\begin{array}{c}\% \text { of } \\
\text { total }\end{array}$ \\
\hline Review & 91 & 100 & 100 \\
Article types & 91 & 100 & 100 \\
Literature review & 51 & 56 & 56 \\
Empirical article & 40 & 44 & 44 \\
Scientific domain & 91 & 100 & 100 \\
Neuropsychology & 82 & 90 & 90 \\
Neurosciences & 25 & 27 & 27 \\
Social sciences & 9 & 10 & 10 \\
Neuropsychiatry & 2 & 2 & 2 \\
Psychology & 1 & 1 & 1 \\
Other & 1 & 1 & 1 \\
\hline
\end{tabular}

Notes. Word count $=$ number of times the word appeared; word weighted percentage $=\%$ of each word in the total of words.
Table 2. Paper variation in terms of diagnosis, population and theoretical models

\begin{tabular}{lccc}
\hline & $\begin{array}{c}\text { No. of } \\
\text { records }\end{array}$ & $\begin{array}{c}\text { \% in the } \\
\text { (sub)category }\end{array}$ & $\begin{array}{c}\% \text { of } \\
\text { total }\end{array}$ \\
\hline Diagnosis & 82 & 90 & 90 \\
Types & 82 & 100 & 90 \\
Consequences & 72 & 88 & 79 \\
Population & 72 & 79 & 79 \\
Children & 26 & 36 & 29 \\
Adolescents and youths & 15 & 21 & 16 \\
Adults & 13 & 18 & 14 \\
Older adults & 4 & 6 & 4 \\
Animals & 4 & 6 & 4 \\
Population country & 2 & 3 & 2 \\
Unspecified & 33 & 46 & 36 \\
Theoretical models & 41 & 45 & 45 \\
Neuropsychological & 30 & 73 & 33 \\
Social & 7 & 17 & 8 \\
Assessment & 6 & 15 & 7 \\
TBI & 6 & 15 & 7 \\
Rehabilitation & 5 & 12 & 5 \\
Awareness & 4 & 10 & 4 \\
Memory & 4 & 10 & 4 \\
Attention & 3 & 7 & 3 \\
Animals & 1 & 2 & 1 \\
Autism spectrum disorders & 1 & 2 & 1 \\
\hline
\end{tabular}

Notes. Word count $=$ number of times the word appeared; word weighted percentage $=\%$ of each word in the total of words.

Of the emerging themes, diagnosis, population, and theoretical models all had a one-layered subdivision (see Table 2).

\section{Diagnosis}

82 articles report types of diagnosis (90\%) and 72 report consequences of the diagnoses $(79 \%)$.

Example of diagnosis:

"Impairment in executive functioning can occur after mild stroke, mild Traumatic Brain Injury, and neurodegenerative disease, and this can have deleterious effects on employment outcomes, occupational functioning, and general quality of life" (Dodson, 2010).

\section{Population}

26 articles report on children (29\%), 15 on adolescents and youths (16\%), 13 on adults (14\%), 4 report on older adults (4\%), 4 on animals (4\%), 2 on population country (2\%), and 33 did not specify the studied population (36\%).

\section{Theoretical Models}

30 articles report on neuropsychological models (33\%), 7 on social models (8\%), 6 on assessment models (7\%), 
Table 3. Paper variation in terms of central nervous system, assessment, and intervention

\begin{tabular}{|c|c|c|c|}
\hline & $\begin{array}{l}\text { No. of } \\
\text { records }\end{array}$ & $\begin{array}{c}\% \text { in the } \\
\text { (sub)category }\end{array}$ & $\begin{array}{l}\% \text { of } \\
\text { total }\end{array}$ \\
\hline Central nervous system & 86 & 95 & 95 \\
\hline (Dys)functions & 85 & 99 & 93 \\
\hline Cognitive (dys)functions & 83 & 98 & 91 \\
\hline Executive function & 72 & 87 & 79 \\
\hline Terminology & 68 & 94 & 75 \\
\hline Components & 32 & 44 & 35 \\
\hline Memory & 36 & 43 & 40 \\
\hline Attention & 31 & 37 & 34 \\
\hline Language & 14 & 17 & 15 \\
\hline Information processing & 12 & 14 & 13 \\
\hline Learning & 11 & 13 & 12 \\
\hline Problem solving & 9 & 11 & 10 \\
\hline Intelligence quotient & 6 & 7 & 7 \\
\hline (Un)awareness & 5 & 6 & 5 \\
\hline Perception & 4 & 5 & 4 \\
\hline Visuospatial functioning & 1 & 1 & 1 \\
\hline Unspecified & 22 & 27 & 24 \\
\hline Behavioral functioning & 23 & 27 & 25 \\
\hline Social skills & 20 & 24 & 22 \\
\hline Physical (dys)functions & 11 & 13 & 12 \\
\hline Other & 47 & 55 & 52 \\
\hline Structures & 33 & 38 & 36 \\
\hline Lesions & 27 & 31 & 30 \\
\hline Types & 26 & 96 & 29 \\
\hline Consequences & 20 & 74 & 22 \\
\hline Assessment & 61 & 67 & 67 \\
\hline Types & 58 & 95 & 64 \\
\hline Neuropsychological & 46 & 79 & 51 \\
\hline Neurological & 15 & 26 & 16 \\
\hline Physical & 10 & 17 & 11 \\
\hline Behavioral & 5 & 9 & 5 \\
\hline Other & 8 & 14 & 9 \\
\hline Instruments & 46 & 75 & 51 \\
\hline Psychometric & 26 & 57 & 29 \\
\hline Neuroimage & 12 & 26 & 13 \\
\hline Other & 10 & 22 & 11 \\
\hline Unspecified & 6 & 13 & 7 \\
\hline Recommendations & 16 & 26 & 18 \\
\hline Limitations & 13 & 21 & 14 \\
\hline Validity & 10 & 16 & 11 \\
\hline Professionals & 5 & 8 & 5 \\
\hline Intervention & 54 & 59 & 59 \\
\hline Types & 49 & 91 & 54 \\
\hline Cognitive rehabilitation & 32 & 65 & 35 \\
\hline Cognitive-behavioral & 8 & 16 & 9 \\
\hline Social & 6 & 12 & 7 \\
\hline Pharmacological & 6 & 12 & 7 \\
\hline Psychological & 3 & 6 & 3 \\
\hline Physical & 2 & 4 & 2 \\
\hline Psychotherapy & 1 & 2 & 1 \\
\hline Unspecified & 19 & 39 & 21 \\
\hline Strategies & 33 & 61 & 36 \\
\hline Recommendations & 32 & 59 & 35 \\
\hline Limitations & 14 & 26 & 15 \\
\hline Professionals & 6 & 11 & 7 \\
\hline Instruments & 3 & 6 & 3 \\
\hline
\end{tabular}

Notes. Word count $=$ number of times the word appeared; word weighted percentage $=\%$ of each word in the total of words.
6 on traumatic brain injury (TBI) models (7\%), 5 on rehabilitation models (5\%), 4 on awareness models (4\%), 4 on memory models (4\%), 3 on attention models (3\%), 1 on animal models $(1 \%)$, and 1 on autism spectrum disorder models (1\%).

Example of neuropsychological models:

"The models put forward to date approach the same reality from a number of different perspectives in some case avoiding certain parts of that reality. In this first part, we review the models and theories of contextual information, structured complex events, working memory, adaptive encoding, Miller and Cohen's integrating theory, and the factorial models of executive control." (Tirapu-Ustárroz et al., 2008a).

The themes central nervous system, assessment, and intervention were subdivided into several child nodes (see Table 3).

\section{Central Nervous System}

85 articles report (dys)functions (93\%), 33 refer to structures involved (36\%), and 27 report on lesions (30\%).

As part of (dys)functions, 83 articles report cognitive (dys)functions (91\%), 23 report on behavioral functioning (25\%), 20 report on social skills (22\%), 11 report on physical (dys)functions (12\%), and 47 refer to other (dys)functions $(52 \%)$.

As part of cognitive (dys)functions, 72 articles (79\%) refer to executive functions, 36 to memory (40\%), 31 to attention (34\%), 14 to language (15\%), 12 to information processing (13\%), 11 to learning (12\%), 9 to problem solving (10\%), 6 to intelligence quotient (7\%), 5 to (un)awareness $(5 \%), 4$ to perception (4\%), 1 to visuospatial functioning (1\%), and $22(24 \%)$ are unspecified.

As part of executive functions, 68 articles report on terminology (75\%) and 32 report on executive function components $(35 \%)$.

Example of terminology:

"The cognitive skills that allow individuals to control and regulate their behavior are called executive functions." (Tirapu-Ustárroz et al., 2008a).

Example of executive function components:

"EF include inhibition of behavior and irrelevant information, nonverbal working memory, verbal working memory, self-regulation of affect, motivation and arousal, planning, decision making, selfmonitoring of the entire solving problem process and self-evaluation of the results of the action taken." (Papazian, Alfonso, \& Luzondo, 2006).

The structures group was not subdivided. The recognized neural substrate of $\mathrm{EF}$ is the (pre)frontal cortex. However, recent articles emphasize the importance of frontal-cortical-subcortical circuits, that is, the importance 
of active and flexible networks and the mediation of dopaminergic neurotransmitters.

Example of structures:

"While lesion studies have demonstrated distinct impairments related to pathology in different frontal regions, it is clear that the frontal lobe syndrome is not restricted to damage to frontal regions. (...) Since no one specific neurologic disorder has a predilection to damage isolated to the frontal lobes, profiles of the dysexecutive syndrome are related to damage to several regions in addition to the frontal lobes." (HannaPladdy, 2007).

As part of the lesions group, 26 papers report types of lesions $(29 \%)$ and 20 refer to consequences of lesions (22\%).

Example of types of lesions:

"In this study, the effectiveness of a group-based attention and problem solving (APS) treatment approach to executive impairments in patients with frontal lobe lesions was investigated.' (Miotto, Evans, de Lucia, \& Scaff, 2009).

Example of consequences:

"Evidence supports visuospatial rehabilitation after right hemisphere stroke, and interventions for aphasia and apraxia after left hemisphere stroke." (Cicerone et al., 2011).

\section{Assessment}

58 articles report on types of assessment (64\%), 46 on instruments used $(51 \%), 16$ on recommendations for assessment (18\%), 13 on assessment limitations (14\%), 10 on assessment validity (11\%), and 5 on professionals involved $(5 \%)$.

As part of assessment types, 46 articles report on neuropsychological assessment (51\%), 15 on neurological assessment $(16 \%), 10$ on physical assessment (11\%), 5 on behavioral assessment (5\%), and 8 on other assessment types $(9 \%)$.

Example of assessment types:

"However, newer and more advanced nonconventional MRI techniques have the capacity to detect invisible brain damage that would otherwise not be detected." (Di Perri, Dalaker, Beyer, \& Zivadinov, 2009).

As part of the instruments group, 26 articles report on psychometric instruments (29\%), 12 on screening instruments $(13 \%), 10$ on other' instrument types $(11 \%)$, and 6 did not specify the instruments $(7 \%)$.

Recommendations, limitations, validity, and professionals were not subdivided.
Examples of recommendations:

"Guided by the International Classification of Functioning, Disability, and Health model (ICF model; Peterson, 2005), we suggest that an important development in the field is moving to formal assessment of executive performance in functional contexts, in addition to more traditional assessment of executive impairment." (Lewis, Babbage, \& Leathem, 2011).

Examples of limitations:

"We discuss the obstacles to accurate measurement of executive control, such as: Its prolonged developmental trajectory; lack of consensus on its definition and whether it is a unitary construct; the inherent complexity of executive control; and the difficulty measuring executive-control functions in laboratory or clinical settings." (Kenworthy, Yerys, Anthony, \& Wallace, 2008).

Example of validity:

"The construct and criterion validities of the parent version of the Behavior Rating Inventory of Executive Function (BRIEF) were evaluated in a sample of 100 6- to 16-year-old children with traumatic brain injury (TBI).” (Donders, DenBraber, \& Vos, 2010).

Examples of professionals:

"Clinical neuropsychologists have adopted numerous (and sometimes conflicting) approaches to the assessment of brain-behavior relationships." (Stuss \& Levine, 2002).

\section{Intervention}

49 articles report on types of interventions (54\%), 33 on intervention strategies $(36 \%), 32$ on recommendations $(35 \%), 14$ on intervention limitations $(15 \%), 6$ on professionals involved $(7 \%)$, and 3 on instruments used for intervention $(3 \%)$.

As part of intervention types, 32 articles report on cognitive rehabilitation (35\%), 8 on cognitive-behavioral intervention (9\%), 6 on social intervention $(7 \%), 6$ on pharmacological intervention $(7 \%), 3$ on psychological intervention $(3 \%), 2$ on physical intervention $(2 \%), 1$ refers to psychotherapy $(1 \%)$, and 19 refer to other interventions (21\%).

Strategies, recommendations, limitations, professionals, and instruments were not subdivided.

Example of strategies to be used in intervention:

"Improving the patient's functioning in the realworld environment must be the major goal, and as such intervention methods require an everyday, real-world contextualization." (Gioia, Kenworthy, \& Isquith, 2010). 
Example of recommendations:

"The review of available evidence points to four major recommendations for the rehabilitation of cognition following brain injury: (1) Access to subacute rehabilitation that is holistic in nature and involves a multidisciplinary or transdisciplinary team to work in an integrated fashion to support physical, cognitive, and social skill retraining is vital to support positive outcome following TBI. (...) (4) Training in the use of supportive devices (either a memory book or more technologically enhanced compensatory devices) to support the individual's daily activities remains central to the independent function of the individual in the community. Though emerging treatments (e.g., virtual reality environments) show relative degrees of promise for inclusion in the rehabilitation of the individual with TBI, these need further evaluation in systematic trials." (Cernich, Kurtz, Mordecai, \& Ryan, 2010).

Example of limitations:

"The benefits of rehabilitation following acquired brain injury (ABI) are all too often disrupted by a lack of engagement in the process, variously attributed to cognitive, emotional and neurobehavioral sequelae, and prominently to impaired selfawareness of deficits." (Medley \& Powell, 2010).

Examples of professionals:

"While individual disciplines are not directly referred to in this paper, input from a comprehensive and coordinated interdisciplinary team is crucial to understanding and reducing the impact of executive deficits on functional performance." (Galvin \& Mandalis, 2009).

Example of instruments:

"The evidence base for the rehabilitation of PM is then considered, focusing on retraining PM, using retrospective memory strategies, problem-solving training, and finally, electronic memory aids." (Fish, Wilson, \& Manly, 2010).

\section{Discussion}

As far as article type is concerned, most of the papers are literature reviews. Because theoretical papers can provide particularly good insights into trends and approaches to a topic of study, they are seminal to the purposes of this work. Accordingly, review was one of the keywords in the search string, possibly directing the search to this particular type of articles. Indicating the nature of the works reviewed, the vast majority of themes appeared under the scientific domains of neuropsychology (specifically, emphasizing the relation between brain and cognitive, emotional, and behavioral systems) and neuroscience (the scientific study of the nervous system). The intersection of neuroscience and cognitive psychology is also reflected, for example, in the combined use of different research methodologies, including hemodynamic approaches (e.g., functional magnetic resonance imaging [fMRI]), that allow the assessment of how brain regulations may relate to behavioral and cognitive measures.

In terms of the central nervous system, a constellation of (dys)functions is reported. They are mostly cognitive (dys)functions, though behavioral, social, motor, and physical (dys)functions also emerge and often occur concomitantly. As expected under the current search, the most frequently mentioned cognitive dysfunctions refer to EF. However, dysfunctions of memory and attention, among others, are also represented. Several works present EF terminology (executive function(s), executive functioning, and executive dysfunction are the most referred terms in the reviewed literature), as well as EF components (subprocesses).

In addition to (dys)functions, different structures of the central nervous system are mentioned. In the case of EF, different subprocesses are associated with specific brain regions, especially different regions in the (pre)frontal cortex: The dysfunctions may result either from direct damage of the (pre)frontal lobes or from the disruption of their connections to other brain regions. In fact, there is growing recognition of the importance of the interconnectivity between different brain regions and of their active role in EF (distributed neuronal system). The articles also refer to types of lesions, namely in (pre)frontal regions of the brain, and their consequences.

Different types of diagnoses appear associated with deficits in specific cognitive functions. Those more closely related with executive dysfunctions are mainly traumatic brain injury (TBI) (e.g., Cicerone et al., 2011), with or without comorbid posttraumatic stress disorder (PTSD), and acquired brain injury (ABI) resulting from nontraumatic brain injury, such as those resulting from stroke (e.g., Simblett \& Bateman, 2011). Other diagnoses associated with cognitive deficits are autism spectrum disorders (ASD) (e.g., Schroeder, Desrocher, Bebko, \& Cappadocia, 2010; Serruya \& Kahana, 2008), cancer, cerebral palsy, and Alzheimer's disease (e.g., Serruya \& Kahana, 2008).

The impact of the diagnoses on cognitive functioning, independence, and quality of life are other emerging topics. In terms of the populations included in the reviewed studies, children are the most referred of all. This result has to do with the methodological approach to the literature in this review, which captures distinct themes that emerge from the various studies (e.g., children, youths, adults, and older adults as distinct categories). It has also to do with those studies in which the population was unspecified. Most studies with unspecified populations actually use adult samples, which suggests that adults are considered the norm in this field, not requiring specification (as opposed to children, for example). If all these studies are added up, the number of references to adults is actually greater than the number of references to children.

Regarding evaluation, the vast majority of studies refer to neuropsychological assessment through standardized 
psychometric measurements administered in controlled environments, such as the laboratory, or in clinical settings. To a lesser degree, neurologic evaluations are also mentioned, through instruments known as neuroimaging techniques, including transcranial magnetic stimulation (TMS), fMRI, positron emission tomography (PET), electroencephalography (EEG), and magnetoencephalography (MEG). Physical and behavioral evaluations also appear. The reviewed studies report limitations of traditional instruments of neuropsychological assessment: For not considering the complex nature of EF, the diversity of subprocesses involved, the specificity of pathologies associated with EF, the necessity of ecological evaluations, the developmental stage of the subjects under evaluation, or the consequences of repeated evaluations, namely learning. Various works propose recommendations to overcome some of the previously mentioned limitations, such as alternative procedures for assessment. Other works propose new instruments and test their validity. The professionals listed as being involved in assessment are clinicians, clinical neuropsychologists, psychologists, or practicing physicians.

In terms of intervention, most treatments pertain to the cognitive rehabilitation type. As in assessment, studies highlight intervention limitations, such as little consideration of rehabilitation for the specificities of pathologies or for the developmental stage in which the intervention occurs, lack of patient involvement in the therapeutic process, in part as a result of patients' limited awareness of their deficits, or methodological issues related with the assessment of intervention effects. Works also include new and promising methodologies that have been used as a means to overcome the limitations of traditional interventions, but which still need further research. In fact, several articles offer recommendations, such as additional development of rehabilitation tools, availability of integrated and holistic services, refinement of rehabilitation techniques, interventions that consider the real-world context, development of transdisciplinary teams, or the use of supportive devices and/or emerging treatments, such as virtual reality environments. The need for further evaluation of these new treatments in systematic trials is also mentioned. The works provide specific strategies and instruments to be used in interventions, aiming to increase the potential for generalization of the learned skills. The professionals cited point to the importance of multi- and interdisciplinary teams.

The theoretical models identified in the literature have attempted to explain cognitive functions (e.g., EF, memory, attention) and disorders (e.g., autism spectrum disorders), and to provide a foundation for assessment or intervention practices (e.g., the basis for motivational interviewing). Although, some references to the models only allow their allocation to a more general category, such as neuropsychological model. Regarding executive function models, the analysis reveals that $\mathrm{EF}$ are viewed as including different processes and subprocesses. EF is conceived as a supervisory capacity for directing more modular or specific processes. Thus, it is viewed as resulting from the combination of cognitive (e.g., working memory, inhibitory control), emotional, and behavioral processes, and this is reflected in the diversity of models. Examples of often mentioned EF models are Mark Ylvisaker's (Ylvisaker, 1998) and Norman and Shallice's models (Norman \& Shallice, 1986).

\section{Limitations}

This review has some limitations. First, it was limited to papers indexed in the ISI Web of Knowledge databases. Although this is today's primary research platform for information on the social sciences and humanities, it is possible that papers in other databases would add further contributions to the results. In the same vein, the search terms used may not have targeted some papers (for example, "neuropsychological patients" or "neuropsychological disease" could be considered as additional search terms in future studies). Additionally, some relevant studies in languages other than English and pertinent papers presented at meetings may have been left out due to the application of exclusion criteria. However, these exclusion procedures were meant to target papers in the primary language of scientific research and to ensure that included papers had undergone a blind peer review process. Second, another limitation of this study had to do with the analysis being conducted on papers' titles and abstracts. However, the goal of this review was to present an exploratory analysis that highlighted the variety of relevant subthemes within this field of research. The broad scope of the field thus identified can now generate more specific questions and topics to be pursued in future studies with a narrower and deeper focus.

\section{Conclusion}

The scientific output on EF conveys the complexity of this topic. The sheer variety of themes associated with EF called for a systematization of the current information around this topic, which constituted the goal of this literature review.

The analysis revealed the current interest in the theme, reflected in the growing number of publications in this area over the years. The following themes emerge in the publications: (1) the different populations with diverse diagnoses, from children to the elderly, or even animals, and the importance of considering the specificities of developmental stages in assessment and intervention practices, (2) theoretical models that attempt to explain the cognitive functions or the proposed interventions, (3) the variety of (dys)functions associated to $\mathrm{EF}$, frequently reflecting structural or functional frontal pathology, (4) the diversity of cognitive processes connected to $\mathrm{EF}$, such as attention and memory, (5) the neuroanatomical correlates of (dys)functions, showing the specialized involvement of the prefrontal cortex, with specific regions mediating different subprocesses and the inclusion of other traditionally less considered regions (e.g., posterior cortical and subcortical regions), suggesting that $\mathrm{EF}$ are mediated by dynamic networks, and that the prefrontal cortex is a heterogeneous neuroanatomical region, (6) the difficulties inherent to 
assessment and intervention, and (7) the proposal of new instruments and strategies for assessment and intervention, and, frequently, recommendations for future work in these domains, revealing the evolution of the theme.

Despite the interesting advances in the last years, EF need more research and further development. Reviewed works suggest future directions for research, pointing to the need of well-controlled studies to include more naturalistic and ecologically valid tasks. More comprehensive assessment of post-injury changes in daily functioning and of the effectiveness of interventions is needed. Focus on the generalization of acquired skills to everyday life, improving promising rehabilitation technique and applying evidence to clinical practice seem to be future trends.

\section{Acknowledgments}

This work was funded by national funds through FCT Portuguese Foundation for Science and Technology (Ref. SFRH/BD/28510/2006), co-funded by FEDER funds through COMPETE - Operational Programme Thematic Factors of Competitiveness.

\section{References}

Baddeley, A. D. (1986). Working memory. New York, NY/ Oxford, UK: Oxford University Press.

Baddeley, A. D., \& Wilson, B. B. (1988). Frontal amnesia and dysexecutive syndrome. Brain and Cognition, 7, 212-230.

Bechara, A., Damasio, H., \& Damasio, A. R. (2000). Emotion, decision making and the orbitofrontal cortex. Cerebral Cortex, 10, 295-307.

Cernich, A. N., Kurtz, S. M., Mordecai, K. L., \& Ryan, P. B. (2010). Cognitive rehabilitation in traumatic brain injury. Current Treatment Options in Neurology, 12, 412-423. doi: 10.1007/s11940-010-0085-6

Cicerone, K. D., Langenbahn, D. M., Braden, C., Malec, J. F., Kalmar, K., Fraas, M., \& Ashman, T. (2011). Evidencebased cognitive rehabilitation: Updated review of the literature from 2003 through 2008. Archives of Physical Medicine and Rehabilitation, 92, 519-530. doi: 10.1016/ j.apmr.2010.11.015

Creswell, J. W. (2003). Research design: Qualitative, quantitative and mixed methods approaches. Thousand Oaks, CA: Sage.

Di Perri, C., Dalaker, T. O., Beyer, M. K., \& Zivadinov, R. (2009). Neuroimaging of cognitive impairments in vascular disease. Neurobiology of Dementia, 84, 49-80. doi: 10.1016/ s0074-7742(09)00404-8

Dodson, M. B. (2010). A model to guide the rehabilitation of high-functioning employees after mild brain injury. Work a Journal of Prevention Assessment \& Rehabilitation, 36, 449-457. doi: 10.3233/wor-2010-1044

Donders, J., DenBraber, D., \& Vos, L. (2010). Construct and criterion validity of the behaviour rating inventory of executive function (BRIEF) in children referred for neuropsychological assessment after paediatric traumatic brain injury. Journal of Neuropsychology, 4, 197-209. doi: 10.1348/174866409x478970

Fish, J., Wilson, B. A., \& Manly, T. (2010). The assessment and rehabilitation of prospective memory problems in people with neurological disorders: A review. Neuropsychological Rehabilitation, 20, 161-179. doi: 10.1080/09602010903126029
Fuster, J. (1997). The prefrontal cortex: Anatomy, physiology, and neuropsychology of the frontal lobes (3rd ed.). New York, NY: Raven Press.

Galvin, J., \& Mandalis, A. (2009). Executive skills and their functional implications: Approaches to rehabilitation after childhood TBI. Developmental Neurorehabilitation, 12, 352-360. doi: 10.1080/17518420903087293

Gioia, G. A., Kenworthy, L., \& Isquith, P. K. (2010). Executive function in the real world: BRIEF lessons from Mark Ylvisaker. Journal of Head Trauma Rehabilitation, 25, 433-439. doi: 10.1097/HTR.0b013e3181fbc272

Hanna-Pladdy, B. (2007). Dysexecutive syndromes in neurologic disease. Journal of Neurologic Physical Therapy, 31, 119-127.

Kenworthy, L., Yerys, B. E., Anthony, L. G., \& Wallace, G. L. (2008). Understanding executive control in autism spectrum disorders in the lab and in the real world. Neuropsychology Review, 18, 320-338. doi: 10.1007/s11065-008-9077-7

Lewis, M. W., Babbage, D. R., \& Leathem, J. M. (2011). Assessing executive performance during cognitive rehabilitation. Neuropsychological Rehabilitation, 21, 145-163. doi: 10.1080/09602011.2010.543867

Lezak, M. D. (1982). The problem of assessing executive functions. International Journal of Psychology, 17, 281-297.

Lezak, M. D. (1987). Relationship between personality disorders, social disturbances and physical disability following traumatic brain injury. Journal of Head Trauma Rehabilitation, 2, 57-69.

Luria, A. R. (1973). The working brain: An introduction to neuropsychology (B. Haigh, Trans.). New York, NY: Basic Books.

Luria, A. R. (1980). Higher cortical functions in man (2nd ed.). New York, NY: Basic Books.

Masterman, D. L., Cummings, J. L. (1997). Frontal-subcortical circuits: The anatomic basis of executive, social and motivated behaviors. Journal of Psychopharmacology, 11, 107-114.

Mateer, C. A. (1999). Executive function disorders: Rehabilitation challenges and strategies. Seminars in Clinical Neuropsychiatry, 4, 50-59.

McCabe, D. P., Roediger, H. L. III, McDaniel, M. A., Balota, D. A., \& Hambrick, D. Z. (2010). The relationship between working memory capacity and executive functioning: Evidence for a common executive attention construct. Neuropsychology, 24, 222-243.

Medley, A. R., \& Powell, T. (2010). Motivational Interviewing to promote self-awareness and engagement in rehabilitation following acquired brain injury: A conceptual review. Neuropsychological Rehabilitation, 20, 481-508. doi: 10.1080/09602010903529610

Miotto, E. C., Evans, J. J., de Lucia, M. C. S., \& Scaff, M. (2009). Rehabilitation of executive dysfunction: A controlled trial of an attention and problem solving treatment group. Neuropsychological Rehabilitation, 19, 517-540. doi: 10.1080/09602010802332108

Norman, D., \& Shallice, T. (1986). Attention to action: Willed and automatic control of behavior. In R. Davidson, G. E. Schwartz, \& D. Shapiro (Eds.), Consciousness and selfregulation: Advances in research and theory (pp. 1-18). New York: Plenum Press.

Papazian, O., Alfonso, I., \& Luzondo, R. J. (2006). Executive function disorders. Revista de Neurologia, 42, S45-S50.

Pennington, B. F. (1997). Dimensions of executive functions in normal and abnormal development. In N. A. Krasnegor, G. R. Lyon, \& P. S. Goldman-Rakic (Eds.), Development of the prefrontal cortex: Evolution, neurobiology, and behavior (pp. 265-281). Baltimore, MD: Brookes.

Peterson, D. B. (2005). International classification of functioning, disability and health: An introduction for rehabilitation psychologists. Rehabilitation Psychology, 50, 15-112. 
Robbins, T. W. (1996). Dissociating executive functions of the prefrontal cortex. Philosophical Transactions of the Royal Society of London (B), 351, 1463-1471.

Roberts, R. J., \& Pennington, B. F. (1996). An interactive framework for examining prefrontal cognitive processes. Developmental Neuropsychology, 12, 105-126.

Schroeder, J. H., Desrocher, M., Bebko, J. M., \& Cappadocia, M. C. (2010). The neurobiology of autism: Theoretical applications. Research in Autism Spectrum Disorders, 4, 555-564. doi: 10.1016/j.rasd.2010.01.004

Serruya, M. D., \& Kahana, M. J. (2008). Techniques and devices to restore cognition. Behavioural Brain Research, 192, 149-165. doi: 10.1016/j.bbr.2008.04.007

Simblett, S. K., \& Bateman, A. (2011). Dimensions of the dysexecutive questionnaire (DEX) examined using Rasch analysis. Neuropsychological Rehabilitation, 21, 1-25. doi: 10.1080/09602011.2010.531216

Stuss, D. T., \& Benson, D. F. (1984). Neuropsychological studies of the frontal lobes. Psychological Bulletin, 95, 3-28.

Stuss, D. T., \& Benson, D. F. (1986). The frontal lobes. New York, NY: Raven Press.

Stuss, D. T., \& Levine, B. (2002). Adult clinical neuropsychology: Lessons from studies of the frontal lobes. Annual Review of Psychology, 53, 401-433.

Tirapu-Ustárroz, J., García-Molina, A., Luna-Lario, P., RoigRovira, T., \& Pelegrín-Valero, C. (2008). Modelos de funciones y control ejecutivo (I) [Models of executive control and functions]. Revista de Neurología, 46, 684-692.

Tirapu-Ustárroz, J., García-Molina, A., Luna-Lario, P., RoigRovira, T., \& Pelegrín-Valero, C. (2008). Modelos de funciones y control ejecutivo (II) [Models of executive control and functions]. Revista de Neurología, 46, 742-750.

Ylvisaker, M. (1998). Traumatic brain injury rehabilitation: Children and adolescents. Boston, MA: Butterworth Heineman. 